Original paper

\title{
Robustness of VMAT and 3DCRT plans toward setup errors in radiation therapy of locally advanced left-sided breast cancer with DIBH
}

\author{
Christer Andre Jensen ${ }^{\mathrm{a}, *}$, Ana María Acosta Roa ${ }^{\mathrm{a}}$, Marie Johansen ${ }^{\mathrm{a}}$, Jo-Åsmund Lund ${ }^{\mathrm{b}, \mathrm{c}}$, \\ Jomar Frengen $^{\mathrm{d}}$ \\ a Department of Oncology, Ålesund Hospital, Ålesund, Norway \\ ${ }^{\mathrm{b}}$ Department of Medicine and Healthcare, Ålesund Hospital, Ålesund, Norway \\ ${ }^{\mathrm{c}}$ Department of Cancer Research and Molecular Medicine, Norwegian University of Science and Technology, Trondheim, Norway \\ d Cancer Clinic, St. Olavs Hospital, Trondheim University Hospital, Trondheim, Norway
}

\section{A R T I C L E I N F O}

\section{Keywords:}

Volumetric modulated arc therapy

VMAT

Deep inspiration breath-hold

Robustness

Perturbed dose

\begin{abstract}
A B S T R A C T
Purpose: The aim of our study was to evaluate and compare the robustness of treatment plans produced using the volumetric modulated arc-therapy (VMAT) and the standard three-dimensional conformal radiotherapy (3DCRT) techniques by estimating perturbed doses induced by localization offsets for deep inspiration breathhold (DIBH) in locally advanced breast cancer radiation therapy.

Methods: Twenty patients with left breast carcinoma requiring radiation therapy were analysed in this planning study. Robust VMAT plans regarding minimum CTV doses and standard 3DCRT plans were produced, and perturbed doses were calculated in accordance with localization values from the weekly offline imaging protocol. Offsets from 5 weeks were summed to a perturbed overall treatment plan. Dose criteria for evaluation were coverage and homogeneity of the target, as well as doses to organs at risk.

Results: VMAT plans resulted in significantly better target coverage compared to 3DCRT, as well as lowered doses to heart and left anterior descending artery, while the perturbed doses were less variable for VMAT than 3DCRT plans. Homogeneity was significantly improved in VMAT plans. The statistical analysis taking all organs into account found that VMAT plans were more robust than 3DCRT to localization offsets $(\mathrm{p}=.001)$. The overall mean setup-deviation for the DIBH-patients was less than $2 \mathrm{~mm}$ in all directions.

Conclusions: VMAT plans were more robust on average than conventional 3DCRT plans for DIBH when localization errors were taken into consideration. The combination of robust VMAT planning and DIBH generally improves the homogeneity and target doses.
\end{abstract}

\section{Introduction}

Radiation therapy (RT) is an integral part of most breast cancer treatments. Irradiation of the breast and regional lymph nodes after breast-conserving surgery in locally advanced breast cancer is standard care, as RT improves local control and overall survival [1]. Radiationinduced heart diseases and cardiovascular events are however well documented side effects of left-sided breast cancer (LSBC) irradiation [2-4]. RT can also lead to secondary malignancies (SM) [5]. Women with breast cancer are at greater risk of developing second primary cancer of the breast as well as of other organs [6]. This may suggest there are risk factors related to the appearance of first primary cancer, and the patient's life culture.

External beam RT for LSBC will to some extent deliver a dose to the heart and lung. Excluding the heart from the field might compromise the dose to the target, but by means of the deep inspiration breath hold (DIBH) technique it is possible to reduce the cardiopulmonary doses while maintaining the prescribed dose to the breast and regional lymph nodes. DIBH has rapidly become the gold standard when treating LSBC patients with radiation therapy, due to the ability to lower doses to the heart $[7,8]$. A deep inspiration breath will raise the chest wall and expand the volume of the lungs, thereby pushing the heart away from the chest wall and increasing the distance between the target and the heart. The method is well established and several groups have previously reported beneficial results using this technique [7,9-11].

Volumetric modulated arc therapy (VMAT) has not been widely used in breast cancer RT, but there are some studies that have investigated the technique for standard use in breast cancer [12-15].

\footnotetext{
* Corresponding author.

E-mail addresses: christer.jensen@helse-mr.no (C.A. Jensen), anaaco@ous-hf.no (A.M.A. Roa), Marie.Johansen@helse-mr.no (M. Johansen), Jo-Asmund.Lund@helse-mr.no (J.-Å. Lund), Jomar.Frengen@stolav.no (J. Frengen).
} 
Three-dimensional conformal radiotherapy (3DCRT) with opposed beams using a flash has been the traditional technique for locally advanced breast cancer. The flash intends to ensure good breast coverage even when intra- or interfractional movement should occur, or in the case of breast swelling or deformation during the RT course. It has been shown that intensity modulated radiation therapy (IMRT) has a better skin-sparing effect than traditional 3DCRT as well as improving the target homogeneity $[16,17]$. Treatment plans using the VMAT technique will typically try to achieve good target coverage of planning target volume (PTV), but intra- and interfraction setup variation as well as changes in breast contour might jeopardize the coverage. VMAT has the advantage of optimizing plans with respect to clinical goals, fast planning and treatment delivery, more homogenous target doses and tailored doses to organs at risk (OAR). Some dosimetry studies have found that VMAT improves target coverage and lowers dose to organs at risk in LSBC compared to 3DCRT [12,13,17-19]. However, since the target is close to the external contour, it is important to be cautious with complex modulations in order to ensure target dose due to lack of buildup and lateral scatter $[20,21]$. Some studies have used a virtual bolus outside the breast contour to ensure coverage, and then removed it before the final dose calculation [22-24]. The term robust in this study refers to the stability of dose when variations in patient setup position occur; less dosimetric variability is said to be more robust [22]. Interand intrafraction variation can to some extent be accounted for with traditional target margins, but there is also another possibility for compensating some of the displacements without significantly increasing the target margins using the shape of the target position uncertainty when optimizing VMAT plans [25]. RayStation (Raysearch Laboratories, Stockholm, Sweden) features a robustness functionality which calculates dose for different scenarios, each of them with the target and the OAR at different possible positions so setup errors can be accounted for while planning criteria are satisfied. This yields to lower dose to normal tissue when compared to using margins generated from overlapping possible target positions, without compromising dose to the target [22].

Two recent studies have reported clinical benefit of including the internal mammary nodes in the clinical target volume, and this might lead way for VMAT as a future gold standard for locoregional patients with internal mammary nodes $[26,27]$. It is crucial that the prescribed and planned dose are consistent, thereby requiring a robust plan in which the delivered dose does not deteriorate throughout the treatment course due to localization offsets and possible changes in the volume of the breast.

The aim of our study was to evaluate the robustness of 3DCRT and VMAT plans. CTV minimum doses were used in robust VMAT optimization. Perturbed dose was calculated for both VMAT and conformal 3DCRT techniques with respect to the actual localization of the patient during the treatment sessions, and the VMAT technique doses were evaluated against the current 3DCRT technique.

\section{Materials and methods}

\subsection{Patient selection and training}

A total of 20 patients referred to Ålesund Hospital with stage pT1cT3N1 left breast carcinoma requiring RT were recruited during the period from June 2014 to February 2015. All patients returned written consent to participate in the Regional Ethics Committee approved protocol. The patients had a median age of 58 (range 25-86) years. In order to be eligible for the DIBH treatment utilizing an in-house laser system, patients had to be able to maintain a stable breath hold for at least $20 \mathrm{~s}$ during training at the CT appointment [28]. There were no limitations regarding age or other diseases. The patients received training for breath hold technique as part of the $30 \mathrm{~min}$ CT acquisition slots. Patients underwent the first part of the DIBH training without visual guided breathing, and their maximum breathing amplitudes were measured. The DIBH-amplitude was chosen as $80 \%$ of maximum inhale amplitude, and a gating window of $\pm 1 \mathrm{~mm}$ was established. Patients were then trained through audio-visual guidance to ensure a stable breath hold during CT-scanning. All patients were immobilized with a WingSTEP $($ (IT-V, Innsbruck, Austria) breast board in the supine position, no tilt was used as the small bore opening of our CT-scanner could not accommodate this. The CT scanner was a 16 slice multi-detector MX8000 Brilliance IDT (Philips Medical Systems, Eindhoven, Netherlands), and images were obtained with $3 \mathrm{~mm}$ slice thickness. Images were transferred to Oncentra Masterplan v 3.4 (Elekta, Crawley, UK) and RayStation v 5.0 (Raysearch, Stockholm, Sweden) treatment planning systems (TPS).

\subsection{Delineation of target and OARs}

The clinical target volume (CTV), heart and left anterior descending artery (LAD), were delineated by a radiation oncologist, while radiation therapists contoured the lungs, spinal canal, contralateral breast and external contour. The CTV included the left breast/chest wall and the supraclavicular and axillary level I-III nodes. The heart and LAD were delineated according to published international guidelines [29]. PTV was automatically generated, derived from CTV with 10/5/5 mm extension in the superior-inferior/anterior-posterior/left-right directions (SI/AP/LR). The first $5 \mathrm{~mm}$ inside the external contour were excluded both from the CTV and the PTV.

\subsection{DCRT treatment planning}

The radiation therapists produced a 3DCRT DIBH treatment plan in accordance to national guidelines based on an in-house protocol, and the duration of the planning process was recorded. Mono-isocentric photon beams with an isocenter below the clavicular head and inside the lung were used. Two wide opposing tangential fields were used; the medial field covered the parts of the target below the isocenter, while the lateral wedged field covered the entire target volume including locoregional periclavicular nodes. Above the isocenter, an anterior wedged field was abutted to the caudal medial field. In addition, some segment beams with low weight were used as aids to achieve dose homogeneity. The clinical goal for target coverage was minimum 95\% of the prescribed dose to the CTV. The national guidelines at the time of inclusion stated that the mean dose of the heart should be under $2 \mathrm{~Gy}$, that less than $35 \%$ of the left lung should receive more than $20 \mathrm{~Gy}$, and that the mean dose to the CTV should be $50 \mathrm{~Gy}$ in 25 fractions (from which only $46 \mathrm{~Gy}$ to the axilla region in 23 fractions). No national guidelines for doses to LAD, spinal canal, contralateral lung or breast were available at the time of the study, so these were planned according to an in house protocol based on the ALARA principle. The modelled machine in the TPS was an Elekta Synergy with a $10 \mathrm{~mm}$ MLC. The treatment plans were calculated with the Collapsed Cone algorithm, and transferred to the record and verify system Mosaiq (Elekta, Crawley, UK) for treatment delivery.

\subsection{VMAT treatment planning}

The VMAT-DIBH treatment plans were generated retrospectively using RayStation. The modelled machine in RayStation was an Elekta VersaHD with a $5 \mathrm{~mm}$ MLC. The VMAT plans were optimized according to the same clinical goals as the original 3D-conformal plans in order to establish if VMAT was suitable for this group of patients, nevertheless, some extra clinical goals, see Table 1 , were necessary and included for the optimization process. To achieve the prescribed dose, two partial $6 \mathrm{MV}$ photon arcs with arc length of $240^{\circ}$, where the start/stop angle was $170^{\circ} / 290^{\circ}$ and collimator angles were $355^{\circ} / 5^{\circ}$. The maximum delivery time was stablished as $90 \mathrm{~s}$.

The robust optimization feature in RayStation is based on the minmax optimization, where a plan is optimized in multiple geometries 
Table 1

Clinical goals used in RayStation.

\begin{tabular}{lll}
\hline Structure & Goals & Weight \\
\hline PTV & 95\% of volume receive 95\% of prescribed dose & High \\
PTV & Maximum dose, 105\% of prescribed dose & High \\
CTV & Uniform dose, prescribed dose & Low \\
Heart & Maximum average 2 Gy & High \\
Left lung & Maximum 35\% of volume receive 20 Gy & Medium \\
Contralateral breast & Max average 3 Gy & Medium \\
External & 3 cm dose Fall-off, distance from high dose to & Medium \\
& low dose (50\% isodose) & \\
\hline
\end{tabular}

and the worst (maximum) objective value from these geometries is used in the objective function. The plan is optimized with isocenter offsets applied in the specified directions, which will define a volume where the plan will be robust $[30,31]$. A robust plan should ensure planning criteria are satisfied when setup errors are accounted for. The VMATDIBH optimization was set to be robust with respect to the distal tangent field edge due to breast movement and volume changes. The plan objective optimized using the robust feature was the minimum dose to the CTV, with a $10 \mathrm{~mm}$ margin in the left-lateral and anterior directions. It is anticipated that coverage in the other directions would be achieved with standard PTV margins. The optimized plan was calculated in five different scenarios.

\subsection{Treatment delivery}

The 3DCRT treatment plans using DIBH were delivered on either Elekta Synergy or Precise machines equipped with 80 leaves MLC and amorphous-silicon flat panel portal imaging systems (iView GT 3.4, Elekta, Crawley, UK). The treatment was performed within a $10 \mathrm{~min}$ treatment slot, only the first session required a double slot. The breathing of the patient was monitored by the radiation therapist, and the beam was manually turned on when the patient was inside the correct gating window [28]. In the case the patient's sternum left the gating window, the radiation therapist had the responsibility to decide if the beam should be interrupted.

All patients followed an offline portal imaging protocol, in which stereoscopic images were acquired on day 1-3 and then weekly. The chest wall and ribs were outlined and used to match the portal image to the digitally reconstructed radiograph from the CT-scan. Localization offset was calculated after the 3rd fraction and systematic errors were corrected. Weekly patient positioning errors of less than $5 \mathrm{~mm}$ were accepted; in case of having deviations over $5 \mathrm{~mm}$ new images were acquired and a new trend was calculated. All portal images were analysed for systematic and random errors in accordance with the formalism proposed by van Herk et al. [32]. The average of the individual systematic setup-up error for the population $(\mu)$, the standard deviation of the individual systematic set-up errors for the population $(\Sigma)$ and the average of the individual random set-up error for the population $(\sigma)$ were calculated. Using van Herk's formalism the margins these patients would need to ensure that $90 \%$ of them obtained $95 \%$ dose coverage to the CTV were estimated. In 3D, the PTV margins are determined using the formula $\mathrm{M}=2.5 \Sigma+0.7 \sigma$.

\subsection{Perturbed treatment plans}

The localization values from the weekly imaging protocol were used to calculate the weekly perturbed dose. The weekly localization values were used as a surrogate of all 5 treatments for the respective week, and the offsets from all 5 weeks were summed to a perturbed overall treatment plan.

\subsection{Data analysis}

The homogeneity index (HI) was calculated as the ratio between the percentage of $\mathrm{D}_{98 \%}$ and $\mathrm{D}_{2 \%}$ for CTV and PTV respectively ( $\mathrm{HI}=\mathrm{D}_{98 \%} /$ $\left.\mathrm{D}_{2 \%}\right)$.

The dosimetric data was analysed using a Wilcoxon signed rank test and considered significant if $\mathrm{p}$ was less than 0.05 . SPSS version 23 (IBM, Armonk, US) was used in the calculations.

\section{Results}

A group of 20 patients underwent in total 500 treatment sessions with the DIBH technique, and weekly offline imaging was performed. The time to produce a 3DCRT plan was in the range of 4.5-33 h, with a median time of $13 \mathrm{~h}$. The relatively long planning time for some patients was due to the strict clinical goals, the complexity of the field setup (median 9 fields, range 8-13 fields), patient body shape and the varying degree of experience of the planners. The median time required for the production of a VMAT plan was $3 \mathrm{~h}$, using the built in optimizer and predefined protocol. The median delivery time for the 3DCRT DIBH technique was $7 \mathrm{~min}$.

\subsection{Reproducibility and stability of DIBH treatment}

A total of 354 localization images from the 20 breast cancer patients were analysed. The overall mean setup-deviation $\mu$ was $0.1 /-0.8$ / $1.8 \mathrm{~mm}$ in $\mathrm{AP} / \mathrm{LR} / \mathrm{SI}$ directions. The systematic error $\Sigma$, and random error $\sigma$, were of the same order of magnitude in all directions $(\Sigma \mathrm{AP}=1.3 \mathrm{~mm} ; \quad \Sigma \mathrm{LR}=1.4 \mathrm{~mm} ; \quad \Sigma \mathrm{SI}=1.4 \mathrm{~mm} ; \quad \sigma \mathrm{AP}=3.1 \mathrm{~mm} ;$ $\sigma \mathrm{LR}=3.8 \mathrm{~mm} ; \sigma \mathrm{SI}=3.3 \mathrm{~mm}$ ).

PTV margins were calculated according to the systematic and random errors. Applying van Herk's formula the margins were found to be $5.5 \mathrm{~mm}$ in the AP direction, $6.3 \mathrm{~mm}$ in the LR direction and $5.7 \mathrm{~mm}$ in the SI direction.

\subsection{Plan robustness}

Dosimetric values for 3DCRT and VMAT plans with and without perturbed doses are shown in Table 2. VMAT plans result in significantly better target coverage. The VMAT technique also significantly lowers mean doses to the heart (14 of 20 perturbed plans) and the LAD (18 of 20 perturbed plans) in comparison to the 3DCRT plans. This comes though at the expense of an increase in low dose bath to both lungs and the contralateral breast. The HI is significantly improved in VMAT plans, and only shows a marginal deterioration in the perturbed VMAT plans.

The average DVHs of the 20 patients for each technique with planned and perturbed dose are shown in Fig. 1. As shown in the bottom right panel of Fig.1, the DVHs for the CTV show worsened coverage for perturbed 3DCRT plans whereas the VMAT plans appear to be very robust against the displacement perturbations.

Fig. 2 shows the mean percentage difference of 3DCRT and VMAT plans after perturbation for dosimetric parameters of the OARs and targets. The statistical analysis taking all organs into account showed a significant dosimetric difference between 3DCRT and VMAT, $\mathrm{p}=.001$. VMAT plans were more robust overall.

\section{Discussion}

In this study, we compared the robustness of 3DCRT and VMAT plans for comprehensive locoregional DIBH radiotherapy of LSBC. We found that the robust optimized VMAT plans provided better target coverage and dose homogeneity than the 3DCRT plans, and that the dose distribution in these VMAT plans was less affected by perturbations when localization offsets of the treatment sessions were accounted for. The treatment planners used several segments to achieve 
Table 2

Dosimetric comparison between 3DCRT and VMAT for planned and perturbed treatment plans.

\begin{tabular}{|c|c|c|c|c|c|c|}
\hline Volume & & 3DCRT & 3DCRT perturbed & VMAT & VMAT perturbed & p-value 3DCRT vs VMAT \\
\hline \multirow[t]{3}{*}{ Heart } & Mean (Gy) & $2.27 \pm 0.72$ & $2.33 \pm 1.02$ & $2.05 \pm 0.31$ & $2.05 \pm 0.33$ & .039 \\
\hline & $\mathrm{V}_{5 \mathrm{~Gy}}(\%)$ & $4.77 \pm 3.37$ & $4.65 \pm 3.59$ & $2.11 \pm 1.80$ & $2.25 \pm 1.98$ & $<.001$ \\
\hline & $\mathrm{V}_{25 \mathrm{~Gy}}(\%)$ & $0.58 \pm 1.14$ & $0.56 \pm 1.12$ & $0.00 \pm 0.00$ & $0.00 \pm 0.00$ & .035 \\
\hline \multirow[t]{2}{*}{ LAD } & Mean (Gy) & $8.71 \pm 6.43$ & $8.12 \pm 6.10$ & $4.58 \pm 1.32$ & $4.73 \pm 1.66$ & .004 \\
\hline & Max (Gy) & $23.67 \pm 12.96$ & $21.41 \pm 12.07$ & $19.04 \pm 9.13$ & $18.46 \pm 9.16$ & .054 \\
\hline \multirow[t]{3}{*}{ Left lung } & Mean (Gy) & $13.27 \pm 2.42$ & $12.88 \pm 2.41$ & $14.48 \pm 1.53$ & $14.11 \pm 1.79$ & .016 \\
\hline & $\mathrm{V}_{5 \mathrm{~Gy}}(\%)$ & $48.34 \pm 6.80$ & $48.14 \pm 6.76$ & $68.38 \pm 8.81$ & $67.88 \pm 9.07$ & $<.001$ \\
\hline & $\mathrm{V}_{20 \mathrm{~Gy}}(\%)$ & $27.34 \pm 6.21$ & $27.04 \pm 6.09$ & $27.42 \pm 3.66$ & $26.87 \pm 3.93$ & .948 \\
\hline \multirow[t]{2}{*}{ Right lung } & Mean (Gy) & $0.97 \pm 0.19$ & $0.96 \pm 0.19$ & $2.48 \pm 1.17$ & $2.46 \pm 1.14$ & $<.001$ \\
\hline & $\mathrm{V}_{5 \mathrm{~Gy}}(\%)$ & $1.55 \pm 1.47$ & $1.44 \pm 1.45$ & $13.49 \pm 12.18$ & $13.37 \pm 12.06$ & $<.001$ \\
\hline \multirow[t]{2}{*}{ Contralateral breast } & Mean (Gy) & $1.23 \pm 0.68$ & $1.17 \pm 0.53$ & $2.63 \pm 0.59$ & $2.60 \pm 0.55$ & $<.001$ \\
\hline & $\mathrm{V}_{5 \mathrm{~Gy}}(\%)$ & $2.07 \pm 3.18$ & $1.93 \pm 2.93$ & $10.56 \pm 6.14$ & $10.50 \pm 5.88$ & $<.001$ \\
\hline Spinal canal & $\mathrm{D}_{1 \mathrm{cc}}(\mathrm{Gy})$ & $26.60 \pm 5.80$ & $23.63 \pm 5.06$ & $16.67 \pm 3.66$ & $15.96 \pm 3.52$ & $<.001$ \\
\hline \multirow[t]{4}{*}{ PTV } & $\mathrm{D}_{98 \%}(\mathrm{~Gy})$ & $44.10 \pm 2.54$ & $41.73 \pm 4.35$ & $46.88 \pm 0.74$ & $44.87 \pm 1.62$ & $<.001$ \\
\hline & $\mathrm{D}_{95 \%}(\mathrm{~Gy})$ & $46.31 \pm 1.21$ & $45.27 \pm 1.86$ & $47.81 \pm 0.38$ & $46.73 \pm 0.82$ & $<.001$ \\
\hline & $\mathrm{D}_{2 \%}(\mathrm{~Gy})$ & $51.90 \pm 0.47$ & $52.66 \pm 1.22$ & $51.68 \pm 0.28$ & $51.52 \pm 0.38$ & $<.001$ \\
\hline & $\mathrm{HI}$ & $0.84 \pm 0.05$ & $0.79 \pm 0.09$ & $0.91 \pm 0.02$ & $0.87 \pm 0.04$ & $<.001$ \\
\hline \multirow[t]{5}{*}{ CTV } & $\mathrm{D}_{98 \%}(\mathrm{~Gy})$ & $46.94 \pm 1.17$ & $46.25 \pm 1.70$ & $48.41 \pm 0.31$ & $48.33 \pm 0.27$ & $<.001$ \\
\hline & $\mathrm{D}_{50 \%}(\mathrm{~Gy})$ & $50.01 \pm 0.28$ & $50.06 \pm 0.75$ & $50.22 \pm 0.08$ & $50.27 \pm 0.16$ & .003 \\
\hline & $\mathrm{D}_{2 \%}(\mathrm{~Gy})$ & $52.50 \pm 0.46$ & $52.86 \pm 1.40$ & $51.65 \pm 0.34$ & $51.56 \pm 0.40$ & $<.001$ \\
\hline & $\mathrm{HI}$ & $0.89 \pm 0.03$ & $0.88 \pm 0.04$ & $0.94 \pm 0.01$ & $0.93 \pm 0.04$ & $<.001$ \\
\hline & Mean (Gy) & $50.14 \pm 0.24$ & $50.12 \pm 0.69$ & $50.19 \pm 0.08$ & $50.18 \pm 0.19$ & .361 \\
\hline External & $\mathrm{D}_{2 \mathrm{cc}}(\mathrm{Gy})$ & $52.63 \pm 2.27$ & $53.53 \pm 1.33$ & $52.40 \pm 0.46$ & $52.06 \pm 0.56$ & .658 \\
\hline
\end{tabular}

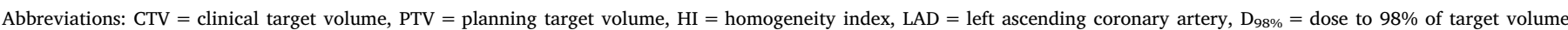

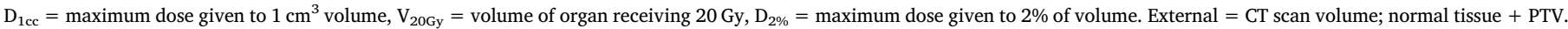

homogeneous doses in 3DCRT plans, and we found that these plans seemed to be more susceptible than VMAT plans to perturbations throughout the treatment sessions. We also found that VMAT plans provided better sparing of heart and LAD, but due to the intrinsic low-
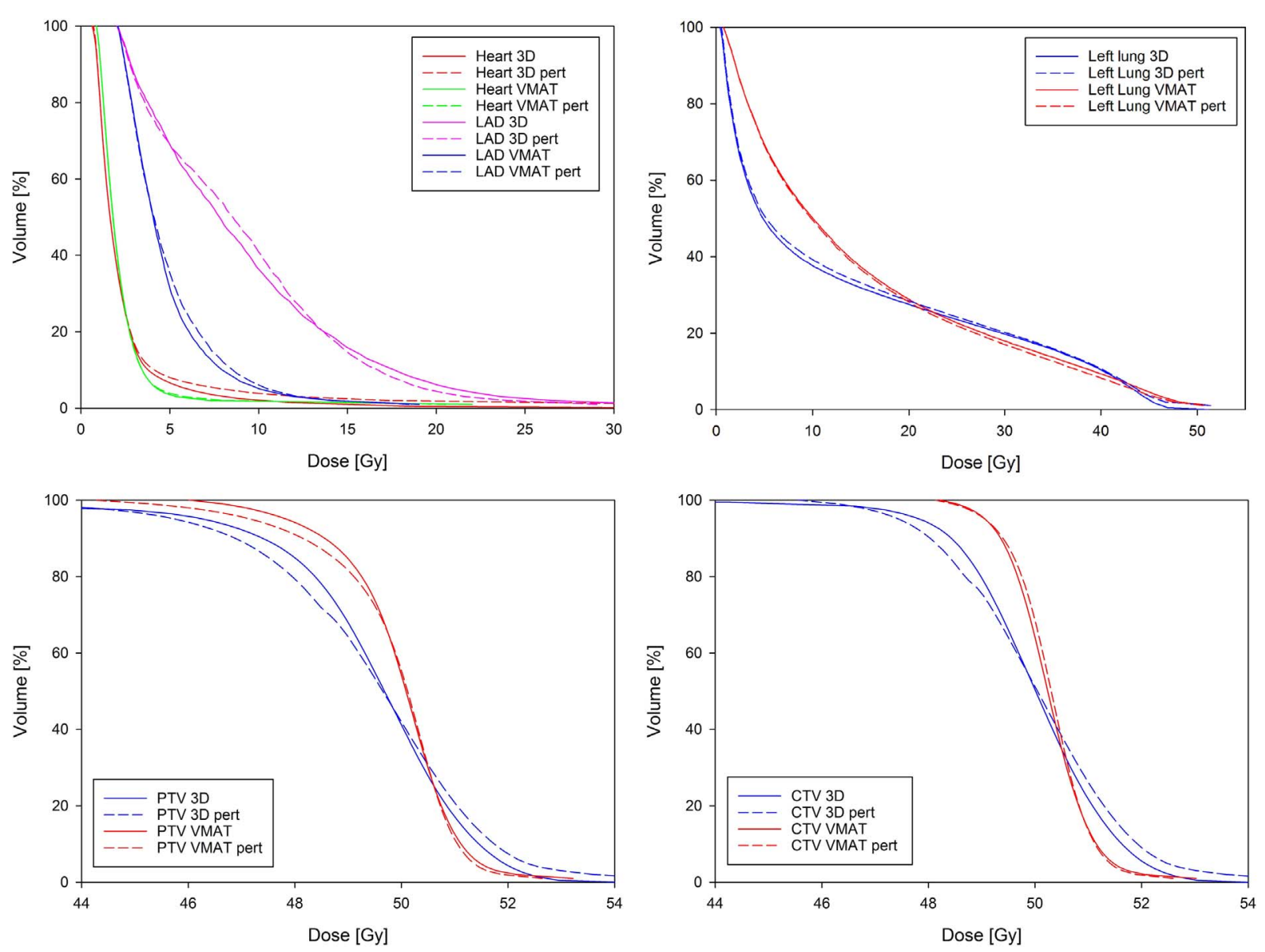

dose bath of the VMAT technique, the contralateral breast and lung received statistically significantly higher doses than the 3D technique, nevertheless, the absolute difference was small and the VMAT plans were considered clinically acceptable. To our knowledge, our study is

Fig. 1. Mean DVHs of 20 patients. Solid lines indicate original treatment plan calculation, while dotted lines represent perturbed doses. 

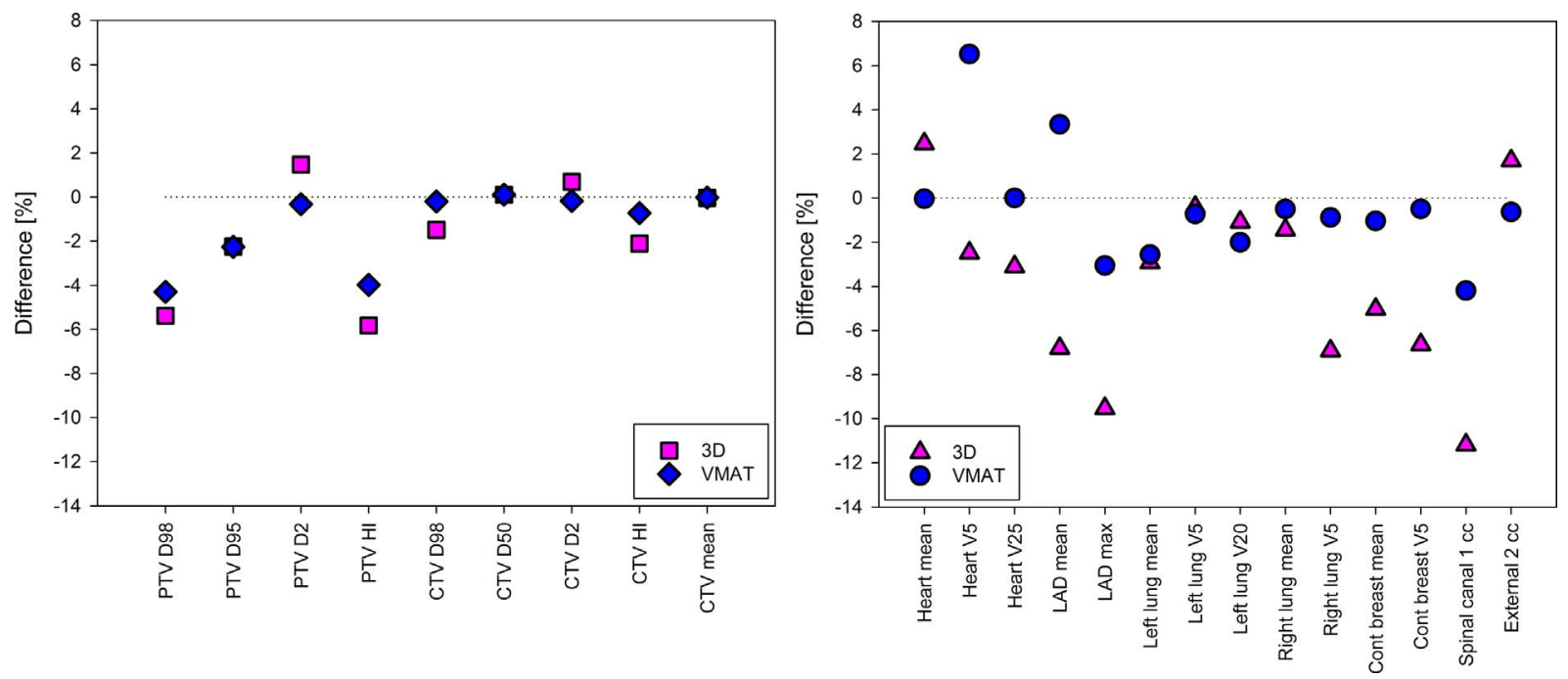

Fig. 2. Mean difference between original and perturbed treatment plans with regards to targets (left) and organs at risk (right).

the first locally advanced DIBH VMAT study that has calculated the perturbed dose distribution based on actual treatment data. We found that the localization offsets had limited influence on the DVHs from the originally planned VMAT plans, most likely due to the effect of the robustness function in RayStation.

The PTV margins should include most of the localization errors. The margin calculation was based upon historical data, and chosen so that $90 \%$ of patients should obtain $95 \%$ dose coverage to the CTV. The results from the 20 patients in our study were within the same order of magnitude as our historical margin data, except from in the SI direction where we calculate a $5.7 \mathrm{~mm}$ margin compared to the larger $10 \mathrm{~mm}$ margins used in the planning. The $\mathrm{D}_{98 \%}$ dose to the CTV (CTV $\mathrm{D}_{98 \%}$ ) is a commonly used clinical parameter to describe the minimum dose to the clinical target. Due to the PTV margin and because the CTV minimum dose was used in the robust VMAT optimization, the CTV $\mathrm{D}_{98 \%}$ was expected to withstand the positioning errors throughout the treatment course. We found that both 3DCRT and VMAT plans sustained a good coverage when the localization offsets were accounted for, but that the CTV $\mathrm{D}_{98 \%}$ dose was 1.47 Gy lower in the 3DCRT plans ( $<$.001) and that this difference increased to $2.08 \mathrm{~Gy}$ in the perturbed plans. Fig. 2 shows the mean difference between the original and perturbed treatment plans with regards to targets and organs at risk. The statistical analysis taking all organs into account showed a significant dosimetric difference $(\mathrm{p}=.001)$, and the VMAT plans were more robust overall than 3DCRT. We did not find any other studies evaluating the influence of localization errors on dose distributions in VMAT plans in current literature. Byrne et al. investigated the robustness functionality in RayStation by measuring doses in a phantom and found that IMRT plans where robust to patient localization errors when this functionality had been used actively in the planning process [22]. The improved VMAT coverage in our study was at the expense of a low dose bath to healthy tissue, thereby delivering a higher volume of low dose to the lungs and to the contralateral breast than in the conventional 3DCRT plans. The mean dose to these organs was significantly higher for VMAT plans than for 3DCRT plans, nevertheless, it was within the dose limit recommendations according to national guidelines [33].

Our study showed that the doses to the heart and LAD for all the reported parameters were significantly lower in the VMAT plans. The doses to the heart for locoregional breast cancer patients are well below what has previously been reported in the literature for VMAT treatment. Osman et al. reported mean heart doses of $4.1 \mathrm{~Gy}$, Swamy et al. reported 9.9 Gy, while Pham et al. reported 5.7 Gy [12-14]. Left sided breast radiation therapy will to some extent irradiate organs at risk and possibly increase the risk of secondary cancer. Darby et al. reported that the rates of major coronary events increased linearly with the mean dose to the heart, and that there seems to be no lower dose threshold [2]. Minimization of cardiac radiation in patients with elevated cardiac risk at baseline can be of great benefit. Cardiac toxicity might also be associated with macrovascular damage to the LAD [34], and we found that the VMAT technique significantly lowered the dose to the LAD and that this dose was robust regarding the perturbed dose. Minimization of dose to heart and LAD is expected to reduce the long-term radiation therapy induced cardiac risk.

The VMAT plans were significantly more homogeneous compared to the 3DCRT plans. Hypofractionated regimes have provided equivalent or lower toxicity and equal cosmetic outcomes in 3DCRT [35]. Mukesh et al. found that improved dose homogeneity translated into superior overall cosmesis as well as a reduced risk of skin telangiectasia [36]. It has been shown in our study, as well as in other studies, that VMAT provides increased dose homogeneity to the target, which has the potential to improve cosmetic outcomes compared to 3DCRT [13,23].

There is no clear consensus about acceptable low doses to the lung, and VMAT typically gives a low-dose bath to both lungs which leads to increased $\mathrm{V}_{5 \mathrm{~Gy}}$ volumes. Allen et al. reported that lung $\mathrm{V}_{5 \mathrm{~Gy}}$ of more than $98 \%$ correlated with pneumonitis. The VMAT plans in our study had in average a $\mathrm{V}_{5 \mathrm{~Gy}}$ of close to $70 \%$ to the left lung, and this is the same number as Haciislamoglu et al. and Osman et al. found while Pham et al. reported a $V_{5 G y}$ of $90 \%[12,14,17]$. The effect of the lowdose bath to the lungs in our VMAT-arm is expected to be very low and not to increase the risk of pneumonitis significantly, nevertheless, a low-dose clinical goal should be included in the optimization (i.e. $\left.\mathrm{V}_{5 \mathrm{~Gy}}<65 \%\right)$.

Burt et al. assessed the risks of SM in breast cancer patients with and without RT in a comprehensive analysis of the SEER database [37]. There was an overall increased risk of SMs for breast cancer (BC) patients with and without RT compared to the general population. They found a statistically significant excess risk of SM in subjects receiving RT, 9.9 vs 34.4 pr 10.000 in non-RT vs RT. The majority of SM was breast SMs. They also found that the risk was most evident in young patients with increasing length of follow-up.

A low average dose to the contralateral breast was one of the priorities during the optimization of the VMAT plans in our study, and the mean dose of the patient population was $2.6 \mathrm{~Gy}$ for this technique. This dose was significantly higher than the contralateral breast dose in our 3DCRT plans, nonetheless, it is in the same order as Johansen et al. and Dumane et al. reported in their VMAT studies [38,39]. Overall survival in breast cancer patients has been significantly improved and therefore the dose to normal tissue should be kept as low as possible. It 
is though possible to lower the dose to the OARs at the expense of the target coverage and homogeneity. Pan et al. performed a systematic review and meta-analysis on the risk of secondary malignancies after partial versus whole breast irradiation [40]. Partial breast irradiation, typically given as intraoperative radiotherapy, gives a very limited dose the organs surrounding the target compared to whole breast external RT. They found no significant difference regarding contralateral breast cancer or secondary non-breast cancers. Aalders et al. investigated contemporary risk of local recurrence (LOR), regional recurrence (RR) and contralateral breast cancer (CBC) in patients who had been previously treated for primary breast cancer by means of external beam RT [41]. They found that rates of LOR/RR/CBC were $2.7 \% / 1.5 \% / 2.9 \%$, respectively. These rates decreased significantly over time in the period 2003-2008. They found that systemic therapy had a protective effect on all three end-points.

The main limitation of our study was that the perturbed dose was calculated in the CT-scans originally acquired for treatment planning instead of CBCTs which now typically can be acquired during the treatment course. Any difference in inhaled volume or change in patient contour was therefore not included in the calculations. It was also assumed that the weekly portal imaging could represent the offsets for the whole week. Another limitation was that the linac model in the TPS for the 3DCRT plans was not the same as for the TPS in which the VMAT plans were generated. This opens a chance for small dosimetric differences due to dissimilar beam models in the TPSs. We recalculated doses between TPSs for 3 patients and found only minor low-dose differences; the largest absolute difference was $+0.4 \%$ for $\mathrm{V}_{5 \mathrm{~Gy}}$ contralateral breast. Dissimilar MLC widths could also be a potential limitation, however a study investigating partial breast irradiation found no significant dosimetric difference to support an advantage of 5 -mm over $10-\mathrm{mm}$ leaf width. [42]

VMAT is still sparsely used in the clinics, and the American Society of Radiation Oncology has not suggested the routinely use of IMRT or VMAT in BC irradiation. This is due to no significant clinical advantages documented yet. There is still no long-term data and any cost-benefit relationship must be modelled as of now. A clinical benefit of including the internal mammary nodes in the clinical target volume will probably lead way for VMAT as a future gold standard for locoregional patients with internal mammary nodes $[26,27]$. The low-dose bath volume has been estimated to increase the risk of secondary cancers, however, there are no studies that have shown as high risk in clinical data as the theoretical models have predicted to date [43]. The increased dose to surrounding tissue could be counterbalanced by the target coverage, increased target homogeneity and decreased volume receiving high doses.

\section{Conclusions}

VMAT plans using the robustness feature of RayStation are less affected in average than conventional 3DCRT plans for LSBC by treatment setup errors. The robustness functionality in RayStation is an effective means to assure sustainable coverage for breast cancer patients, and is comparable to the flash overshoot in traditional 3D plans for moderate displacements. The combination of robust VMAT planning and DIBH improves on average the homogeneity and target doses. It is imperative that clinical goals are used to avoid low-dose bath to organs at risk as much as possibly achievable. Further studies evaluating the clinical outcome and long-term risk should be addressed, and as now, it is necessary to balance the risk of low-dose bath against the clinical advantages.

\section{Acknowledgements}

This work was supported by the Liaison Committee between the Central Norway Regional Health Authority (RHA) and the Norwegian University of Science and Technology (NTNU).

\section{References}

[1] Veronesi U, Cascinelli N, Mariani L, Greco M, Saccozzi R, Luini A, et al. Twenty-year follow-up of a randomized study comparing breast-conserving surgery with radical mastectomy for early breast cancer. New Engl J Med 2002;347:1227-32.

[2] Darby SC, Ewertz M, McGale P, Bennet AM, Blom-Goldman U, Bronnum D, et al. Risk of ischemic heart disease in women after radiotherapy for breast cancer. New Eng J Med 2013;368:987-98.

[3] Henson KE, McGale P, Taylor C, Darby SC. Radiation-related mortality from heart disease and lung cancer more than 20 years after radiotherapy for breast cancer. $\mathrm{Br}$ J Cancer 2013;108:179-82.

[4] Boero IJ, Paravati AJ, Triplett DP, Hwang L, Matsuno RK, Gillespie EF, et al. Modern radiation therapy and cardiac outcomes in breast cancer. Int J Radiat Oncol Biol Phys 2016;94:700-8.

[5] Suit H, Goldberg S, Niemierko A, Ancukiewicz M, Hall E, Goitein M, et al. Secondary carcinogenesis in patients treated with radiation: a review of data on radiationinduced cancers in human, non-human primate, canine and rodent subjects. Radiat Res 2007;167:12-42.

[6] Marcu LG, Santos A, Bezak E. Risk of second primary cancer after breast cancer treatment. Eur J Cancer Care (Engl) 2014;23:51-64.

[7] Pedersen AN, Korreman S, Nystrom H, Specht L. Breathing adapted radiotherapy of breast cancer: reduction of cardiac and pulmonary doses using voluntary inspiration breath-hold. Radiother Oncol J Eur Soc Ther Radiol Oncol 2004;72:53-60.

[8] Hjelstuen MH, Mjaaland I, Vikstrom J, Dybvik KI. Radiation during deep inspiration allows loco-regional treatment of left breast and axillary-, supraclavicular- and internal mammary lymph nodes without compromising target coverage or dose restrictions to organs at risk. Acta Oncol 2012;51:333-44.

[9] Vikstrom J, Hjelstuen MH, Mjaaland I, Dybvik KI. Cardiac and pulmonary dose reduction for tangentially irradiated breast cancer, utilizing deep inspiration breathhold with audio-visual guidance, without compromising target coverage. Acta Oncol 2011:50:42-50.

[10] Nissen HD, Appelt AL. Improved heart, lung and target dose with deep inspiration breath hold in a large clinical series of breast cancer patients. Radiother Oncol 2013;106:28-32

[11] Swanson T, Grills IS, Ye H, Entwistle A, Teahan M, Letts N, et al. Six-year experience routinely using moderate deep inspiration breath-hold for the reduction of cardiac dose in left-sided breast irradiation for patients with early-stage or locally advanced breast cancer. Am J Clin Oncol 2013;36:24-30.

[12] Osman SO, Hol S, Poortmans PM, Essers M. Volumetric modulated arc therapy and breath-hold in image-guided locoregional left-sided breast irradiation. Radiother Oncol J Eur Soc Ther Radiol Oncol 2014;112:17-22.

[13] Swamy ST, Radha CA, Kathirvel M, Arun G, Subramanian S. Feasibility study of deep inspiration breath-hold based volumetric modulated arc therapy for locally advanced left sided breast cancer patients. Asian Pac J Cancer Prev 2014:15:9033-8.

[14] Pham TT, Ward R, Latty D, Owen C, Gebski V, Chojnowski J, et al. Left-sided breast cancer loco-regional radiotherapy with deep inspiration breath-hold: does volumetric-modulated arc radiotherapy reduce heart dose further compared with tangential intensity-modulated radiotherapy? J Med Imaging Radiat Oncol 2016;60:545-53.

[15] Boman E, Rossi M, Haltamo M, Skyttä T, Kapanen M. A new split arc VMAT technique for lymph node positive breast cancer. Physica Med Eur J Med Phys 2016;32:1428-36.

[16] Jo IY, Kim S-W, Son SH. Dosimetric evaluation of the skin-sparing effects of 3 dimensional conformal radiotherapy and intensity-modulated radiotherapy for left breast cancer. Oncotarget 2016;5.

[17] Haciislamoglu E, Colak F, Canyilmaz E, Dirican B, Gurdalli S, Yilmaz AH, et al. Dosimetric comparison of left-sided whole-breast irradiation with 3DCRT, forwardplanned IMRT, inverse-planned IMRT, helical tomotherapy, and volumetric arc therapy. Phys Med 2015;31:360-7.

[18] Tyran M, Mailleux H, Tallet A, Fau P, Gonzague L, Minsat M, et al. Volumetricmodulated arc therapy for left-sided breast cancer and all regional nodes improves target volumes coverage and reduces treatment time and doses to the heart and left coronary artery, compared with a field-in-field technique. J Radiat Res 2015;56:927-37.

[19] Bodez V, Duqueyroix F, Fourvel M, Pradon V, Roux V, Mége A, et al. Conformal segmented mono-isocentric radiotherapy vs. volumetric modulated arctherapy in senology. Physica Med Eur J Med Phys 2016;32:375.

[20] Thomas SJ, Hoole ACF. The effect of optimization on surface dose in intensity modulated radiotherapy (IMRT). Phys Med Biol 2004;49:4919-28.

[21] Nguyen TB, Hoole AC, Burnet NG, Thomas SJ. The optimization of intensity modulated radiotherapy in cases where the planning target volume extends into the build-up region. Phys Med Biol 2009;54:2511-25.

[22] Byrne M, Hu Y, Archibald-Heeren B. Evaluation of RayStation robust optimisation for superficial target coverage with setup variation in breast IMRT. Australas Phys Eng Sci Med 2016;39:705-16.

[23] Viren T, Heikkila J, Myllyoja K, Koskela K, Lahtinen T, Seppala J. Tangential volumetric modulated arc therapy technique for left-sided breast cancer radiotherapy. Radiat Oncology 2015;10:79.

[24] Monti A, Brambilla MG, Sarno L, Torresin A, Maldera A, Ferrari MB, et al. Bolus in VMAT breast treatment. Physica Med Eur J Med Phys 2016;32:45.

[25] Fredriksson A, Forsgren A, Hårdemark B. Maximizing the probability of satisfying the clinical goals in radiation therapy treatment planning under setup uncertainty. Med Phys 2015;42:3992-9.

[26] Poortmans PM, Collette S, Kirkove C, Van Limbergen E, Budach V, Struikmans H, 
et al. Internal mammary and medial supraclavicular irradiation in breast cancer. New Eng J Med 2015;373:317-27.

[27] Thorsen LB, Offersen BV, Dano H, Berg M, Jensen I, Pedersen AN, et al. DBCG-IMN: a population-based cohort study on the effect of internal mammary node irradiation in early node-positive breast cancer. J Clin Oncol 2016;34:314-20.

[28] Jensen CA, Skottner N, Frengen J, Lund J-Å. Development of a deep inspiration breath-hold system for radiotherapy utilizing a laser distance measurer. J Appl Clin Med Phys 2017;18:260-4.

[29] Feng M, Moran JM, Koelling T, Chughtai A, Chan JL, Freedman L, et al. Development and validation of a heart atlas to study cardiac exposure to radiation following treatment for breast cancer. Int J Radiat Oncol Biol Phys 2011;79:10-8.

[30] Fredriksson A, Forsgren A, Hardemark B. Minimax optimization for handling range and setup uncertainties in proton therapy. Med Phys 2011;38:1672-84.

[31] Fredriksson A, Bokrantz R. A critical evaluation of worst case optimization methods for robust intensity-modulated proton therapy planning. Med Phys 2014:41:081701.

[32] van Herk M. Errors and margins in radiotherapy. Semin Radiat Oncol 2004; 14:52-64.

[33] Norwegian Breast Cancer Group, http://www.nbcg.no. [accessed 27 Dec 2016].

[34] Marks LB, Zagar TM, Kaidar-Person O. Reassessing the time course for radiationinduced cardiac mortality in patients with breast cancer. Int J Radiat Oncol Biol Phys 2017;97:303-5.

[35] Valle LF, Agarwal S, Bickel KE, Herchek HA, Nalepinski DC, Kapadia NS Hypofractionated whole breast radiotherapy in breast conservation for early-stage breast cancer: a systematic review and meta-analysis of randomized trials. Breast Cancer Res Treat 2017;162:409-17.
[36] Mukesh MB, Barnett GC, Wilkinson JS, Moody AM, Wilson C, Dorling L, et al. Randomized controlled trial of intensity-modulated radiotherapy for early breast cancer: 5-year results confirm superior overall cosmesis. J Clin Oncol 2013;31:4488-95.

[37] Burt LM, Ying J, Poppe MM, Suneja G, Gaffney DK. Risk of secondary malignancies after radiation therapy for breast cancer: comprehensive results. Int J Radiat Oncol Biol Phys 2015;93:S107-8.

[38] Johansen S, Cozzi L, Olsen DR. A planning comparison of dose patterns in organs at risk and predicted risk for radiation induced malignancy in the contralateral breast following radiation therapy of primary breast using conventional, IMRT and volumetric modulated arc treatment techniques. Acta Oncol 2009;48:495-503.

[39] Dumane VA, Hunt MA, Green S, Lo Y-C, Bakst RL. Dosimetric comparison of volumetric modulated arc therapy, static field intensity modulated radiation therapy, and 3D conformal planning for the treatment of a right-sided reconstructed chest wall and regional nodal case. J Radiother 2014;2014:1-12.

[40] Pan X-B, Huang S-T, Jiang Y-M, Ma J-L, Zhu X-D. Secondary malignancies after partial versus whole breast irradiation: a systematic review and meta-analysis. Oncotarget 2016;7:71951-9.

[41] Aalders KC, van Bommel AC, van Dalen T, Sonke GS, van Diest PJ, Boersma LJ, et al. Contemporary risks of local and regional recurrence and contralateral breast cancer in patients treated for primary breast cancer. Eur J Cancer 2016;63:118-26.

[42] Height FJ, Kron T, Willis D, Chua BH. Impact of MLC leaf width on the quality of the dose distribution in partial breast irradiation. Med Dosim 2012;37:37-41.

[43] Chargari C, Goodman KA, Diallo I, Guy J-B, Rancoule C, Cosset J-M, et al. Risk of second cancers in the era of modern radiation therapy: does the risk/benefit analysis overcome theoretical models? Cancer Metastasis Rev 2016;35:277-88. 